\title{
Prehabilitation in thoracic surgery
}

\section{David Sanchez-Lorente ${ }^{1}$, Ricard Navarro-Ripoll ${ }^{2}$, Rudith Guzman ${ }^{1}$, Jorge Moises ${ }^{3}$, Elena Gimeno ${ }^{3}$, Marc Boada $^{1}$, Laureano Molins ${ }^{1}$}

${ }^{1}$ General Thoracic Surgery Department, Clinical Respiratory Institute, ${ }^{2}$ Department of Anesthesiology, ${ }^{3}$ Department of Pneumology, Clinical Respiratory Institute, Hospital Clínic of Barcelona, Barcelona, Spain

Contributions: (I) Conception and design: D Sanchez-Lorente; (II) Administrative support: D Sanchez-Lorente, R Guzman, J Moises, E Gimeno, M Boada, L Molins; (III) Provision of study materials or patients: D Sanchez-Lorente, R Navarro; (IV) Collection and assembly of data: All authors; (V) Data analysis and interpretation: D Sanchez-Lorente; (VI) Manuscript writing: All authors; (VII) Final approval of manuscript: All authors.

Correspondence to: David Sanchez-Lorente, MD, PhD. General Thoracic Surgery Department, Clinical Respiratory Institute, Hospital Clinic of Barcelona, 08036, Barcelona, Spain. Email: dsanche1@clinic.cat.

\begin{abstract}
Surgical resection remains the best treatment option for patients with early stage of non-small cell lung cancer (NSCLC). However, it may be responsible of postoperative complication and mortality, especially in patients with impaired pulmonary function. Enhanced recovery after surgery (ERAS) programs have been focused mainly in minimal invasive surgery approach during lung resection and respiratory rehabilitation after surgery. Preoperative exercise-based intervention (prehabilitation) has demonstrated reduction of morbi-mortality in other surgeries but in thoracic surgery continues to be under discussion. Cardio-pulmonary exercise test (CPET) is the gold standard technique to predict postoperative morbimortality. The implementation of a preoperative respiratory rehabilitation could optimize patient's physical capacity before surgery and improve outcomes and enhance recovery. The aim of this systematic review of the literature is to identify the effectiveness and safety of prehabilitation programs in thoracic surgery, the type of exercise and its duration, and the group of patients with best benefit. Prehabilitation is a safe intervention without side effects in patients. High-intensity interval training (HIT) with duration of 2 to 6 weeks seems to be the best exercise programme in a prehabilitation intervention but it exists heterogeneity in terms of intensity and duration. Prehabilitation increase exercise capacity and significantly enhances pulmonary function. But the reduction of postoperative complication and mortality has not been clearly demonstrated. Different criteria selection, type of intervention and small sample size, in addition to no randomization, could justify disparate results. It seems that not all patients can benefit from prehabilitation and it could be indicated only in patients with impaired lung function. Further randomized clinical trials with enough patients, correct duration of HIT ( 2 to 6 weeks) and focused in COPD patients are needed to clarify the suitability of prehabilitation. Meanwhile, safety of prehabilitation and good results of some studies support this intervention in high-risk patients.
\end{abstract}

Keywords: Prehabilitation; lung cancer; lung resection; enhanced recovery after surgery (ERAS); fast track; respiratory physiotherapy; rehabilitation; preconditioning

Submitted Jun 18, 2018. Accepted for publication Aug 01, 2018.

doi: $10.21037 /$ jtd.2018.08.18

View this article at: http://dx.doi.org/10.21037/jtd.2018.08.18

\section{Introduction}

Lung cancer continues to be the most common and deadly malignancy in developed countries. Anatomic resection with radical lymph node dissection is the only curative option for this pathology. However, not all patients could be operated. Indication for lung resection depends not only upon the histological characteristics of the tumour but also the preoperative staging according to the classification of malignant tumours TNM (1-3). 
Also, it is necessary to assess the surgical risk, which is influenced mainly by co-morbidities and cardiopulmonary function. Approximately $73 \%$ of men and $53 \%$ of women are diagnosed with chronic obstructive pulmonary disease (COPD) along with lung cancer (4). In patients suitable for anatomically resectable lung cancer almost $37 \%$ of them are considered inoperable due to severe pulmonary function impairment (5). The surgical morbidity and mortality rates for these patients at a high risk of perioperative complications are $83 \%$ and $33 \%$ respectively (6). Enhanced recovery after surgery (ERAS) programs have arisen as a way to decrease the morbidity and mortality rates in patients with resectable lung cancer. They include different strategies: (I) modern surgical treatment including minimal invasive surgery as video-assisted thoracoscopic surgery (VATS) has increased in past decades worldwide with a significant survival benefit in individuals with $\operatorname{NSCLC~}(7,8)$ and (II) postoperative rehabilitation have demonstrate to be safe and well-tolerated, and is associated with improvements in physical and physiological outcomes $(9,10)$. A Cochrane systematic review from 2012 highlights that exercise in patients with different type of cancers may have beneficial effects on quality of life (11), but what are the effects of a preoperative exercise program (prehabilitation) for patients with resectable lung cancer? Could prehabilitation optimize the physical status and overall medical stability before surgery and reduce postoperative morbidity? And, could these preconditioning exercises increase the percentage of operable cases by improving the physical status of a patient who was initially considered inoperable due to severe pulmonary function impairment? This review article tries to answer these questions discussing the literature evidence of prehabilitation effects in patients awaiting lung cancer surgery.

\section{Operability evaluation}

Although resection is the best option for treating patients with NSCLC, abnormal pulmonary function still occurs in patients with potentially resectable tumours. The benefit of surgery in these patients with an increased risk of immediate and long-term postoperative complications must be balanced against the reduced survival if we decide of nonsurgical treatment. So, preoperative functional evaluation is deemed necessary. Low postoperative predictive (ppo) values of forced expiratory volume in first second $\left(\mathrm{FEV}_{1}\right)$ and diffusion capacity for carbon monoxide (DLCO) are used to exclude patients from surgery as both have been reported to be independent predictors of post-operative morbidity and mortality after lung resection. According to the joint ERS/ESTS clinical practice guidelines (12) patients with a ppoFEV 1 and ppoDLCO $>80 \%$ are considered to be at low risk and could be operated with an acceptable postoperative risk. However, further assessment is necessary in patients were either one of these parameters are decreased. The ppoFEV1 and ppoDLCO cut-off for higher risk is set at $30 \%$. Exercise testing, mainly cardiopulmonary exercise test (CPET), is the gold standard technique to predict postoperative complications and mortality, especially for borderline patients. Exercise capacity and maximum oxygen uptake $\left(\mathrm{VO}_{2} \max \right)$ parameters are determined by these exercise testing. A $\mathrm{VO}_{2} \max >20 \mathrm{~mL} / \mathrm{kg} / \mathrm{min}$ or at

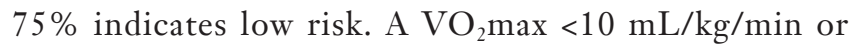
$35 \%$ predicted indicates a high risk of mortality which may be higher than $10 \%$ and practically contraindicate the surgery. If $\mathrm{VO}_{2} \max$ is between 10 and $20 \mathrm{~mL} / \mathrm{kg} / \mathrm{min}$ or $35-75 \%$ the postoperative risk is moderate and ppoFEV1 and ppoDLCO need to be considered to recommend or not resection (cut-off 30\%).

American College of Chest Physicians (ACCP) provided a guideline in 2013 with similar ERS/ESTS indications. ACCP consider patients with a ppoFEV1 and ppoDLCO $>60 \%$ to be at low risk and advice to perform CPET after a negative stair climb test or shuttle walk test in patients with ppoFEV $_{1}$ or ppoDLCO $<60 \%$ and both $>30 \%$. Patients with a ppoFEV1 or ppoDLCO $<30 \%$ need a CPET directly to evaluate the risks with same categories as ERS/ESTS guidelines.

\section{Prehabilitation in an ERAS program}

ERAS program has demonstrated, especially in colorectal surgery, to reduce postoperative complications and length of stay (13). The development of an ERAS program in thoracic surgery has received less attention; nonetheless, its introduction has shown to decrease postoperative complications $(14,15)$. Generally, ERAS interventions are divided into the preoperative, intraoperative and postoperative phases. Intraoperative phase in thoracic surgery has been focused in optimal analgesic technique and minimal invasive surgery (VATS) where possible. In postoperative period, a pain management, early mobilization, early oral intake, and prophylaxis of nausea and vomiting are the main targets. And finally, the preoperative intervention, which probably has been introduced later in thoracic ERAS programs and limited to 
nutritional assessment and treatment, anaemia correction and smoking cessation advice.

As we have previously mentioned the degree of risk for postoperative complications is determined mainly by the lung function, calculated by the predicted post-operative $\mathrm{FEV}_{1}$ and DLCO, and when lung function is affected cardiopulmonary exercise test calculated by climbing test or shuttle walk test (low technology exercise test), or by maximal oxygen consumption $\left(\mathrm{VO}_{2} \mathrm{max}\right)$. Therefore, it seems reasonable to assume that if we are able to improve the lung function of the patient or his capacity to exercise, on the one hand we can decrease the risk for post-operative complications, and on the other hand patients in high risk of complications and mortality, considered inoperable due to cardiopulmonary impairment, could be operated after an intervention previous to the surgery. In our opinion a prehabilitation intervention within an ERAS program should not be just limited to advices and smoking cessation. A more proactive intervention is needed with physical exercise.

\section{Prehabilitation effects}

At the beginning of this century, in a relatively short period of time, pulmonary rehabilitation has become recognized as a cornerstone in the management of patients with COPD (16). Rehabilitation programs including physical exercise and education have showed shorts-term benefits from even brief 2 -week inpatients programs, however conventional rehabilitation programs are generally 8-12 weeks in duration. The evidence for improvement in exercise endurance, dyspnea, functional capacity, and quality of life is stronger for rehabilitation than for almost any other therapy in COPD, and documentation of its favourable effects is increasing. Given the high percentage of COPD in patients diagnosed of lung cancer it is expected we could obtain the benefits of rehabilitation, showed in COPD patients, in patients with lung cancer and some grade of COPD. In fact, pulmonary rehabilitation is so important that it is considered a necessary component before and after lung volume reduction surgery and lung transplantation (17).

\section{Which type of physical exercise?}

Traditionally, endurance training (ET) has been the preferred method of training to obtain changes and improve exercise resistance in rehabilitation, showing improvement in aerobic performances as a result of blood volume expansion, higher cardiac output and enhanced muscle oxygen extraction (18). Endurance training entails moderate intensity efforts during daily sessions lasting 60 to $120 \mathrm{~min}$ and scheduled over a prolonged period of 6 to 12 weeks. And this "long" period of time is one of the limitations to apply this training in patients waiting for a lung resection. The delay from resection indication to surgery for lung cancer was within the maximal waiting time of 4 weeks recommended way some societies (19). Alternatively, high-intensity interval training (HIT) has long been employed by non-medical intention as an equally effective training method to improve aerobic performance measures (20). Although there is no universal definition, HIT generally refers to repeated sessions of relatively brief intermittent exercise, often performed at a high intensity close to $\mathrm{VO}_{2}$ max during few seconds with a previous warmup period, pauses between "peak-exercise" of minutes, and a cooled down. The high intensity exercise is repeated four to six times per training session, with three training sessions performed each week for two to six weeks (21). Dunham and Harms (22) in 2012 published their results in a randomized trial with healthy subjects (untrained) assigned to an ET group or a HIT group and compare to determine the effects of HIT on the pulmonary system. The results of this study suggest that both ET and HIT are effective in increasing inspiratory muscle strength with HIT offering a time-efficient alternative to ET in improving aerobic capacity and performance. Hwang et al. (23) evaluated the effects of HIT in patients with NSCLC comparing the results with a control group. The group of patients submitted to HIT showed an increased $\mathrm{VO}_{2} \max$; these changes were associated with improvements in circulatory, respiratory and muscular functions. Also the HIT group had less dyspnea and favourable lower fatigue than baseline.

So, considering the similar beneficial effects of HIT than ET in a short period of time, this physical exercise training system seems to be the best option in a prehabilitation program, in order to not delay the surgery. However, the work rate needs to be adjusted by the physiotherapist on each patient and session to target near $\mathrm{VO}_{2}$ max, but not too intensive to avoid cardio-respiratory adverse events.

\section{Who could benefit from a prehabilitation?}

Prehabilitation is not a new concept in thoracic surgery, in a selective group of patients pulmonary rehabilitation has been used routinely for high-risk patients undergoing 
thoracic surgery, including lung transplantation and lung reduction volume surgery, to increase their functional capacity and prevent postoperative clinical and physical deterioration $(24,25)$. However, the benefit of an exercise intervention in lung cancer population continues to be unclear. And in case to demonstrate to be beneficial is this benefit the same king of patients?

In 2005, Sekine et al. (26) prospectively enrolled 22 lung cancer patients with COPD (FEV1/FVC $\leq 70 \%$ and more than $50 \%$ ) who underwent lobectomy after a pulmonary rehabilitation program and compare postoperative pulmonary complications of this group of patients with a historical control group of 60 patients with lung cancer who fulfilled the same criteria but did not receive rehabilitation. A statistical significance lower incidence of postoperative pulmonary complications and shorter hospital stay were detected in rehabilitation group. These results promoted new investigation and Jones et al. (27) investigate the effects of preoperative exercise training on cardiorespiratory fitness in patients undergoing thoracic surgery for malignant lung lesion. In a series of 25 patients with lung cancer structured exercise training until surgical resection was provided. Participants underwent cardiopulmonary exercise testing, 6-minute walk (6 MW), and pulmonary function testing at baseline, immediately before and 30 days after surgical resection. That patients who attended to $\geq 80 \%$ of prescribed sessions increased $\mathrm{VO}_{2} \max$ by $3.3 \mathrm{~mL} / \mathrm{kg} / \mathrm{min}(\mathrm{P}=0.006)$ and $6 \mathrm{MW}$ by 49 meters $(\mathrm{P}=0.013)$. Similar results were observed by Bobbio et al. (28) in a prospective observational study including 12 patients with stage I or II NSCLC and COPD on preoperative pulmonary function test with a $\mathrm{VO}_{2} \max \leq 15 \mathrm{~mL} / \mathrm{kg} / \mathrm{min}$. In these patients a pulmonary rehabilitation programme lasting 4 weeks was performed. After rehabilitation patients underwent a new functional evaluation prior to surgery, and the mean increase in $\mathrm{VO}_{2}$ max proved to be at $2.8 \mathrm{~mL} / \mathrm{kg} / \mathrm{min}$.

In a recent study, Tarumi et al. (29) studied the effects of pulmonary rehabilitation in a special group of patients with lung cancer, patients who need an induction chemoradiotherapy before lung resection, and rehabilitation was done during induction treatment. Eighty-two consecutive patients participated in the study. Pulmonary rehabilitation was started at the same time as the induction chemoradiotherapy for an average of 10 weeks, and standard respiratory function test were performed before and after induction treatment. All patients experienced an increase in $\mathrm{FVC}(+6.4 \%, \mathrm{P}=0.0096)$ and $\mathrm{FEV} 1(+10.4 \%$,
$\mathrm{P}<0.001)$. This increase was more significant in patients with respiratory impairment (FVC $<80 \%$ predicted or FEV1/FVC < 70\%) with significant improvement in FVC $+13.9 \%(\mathrm{P}=0.0025)$ and FEV1 $+22.5 \%(\mathrm{P}<0.0001)$.

The Jones, Bobbio and Tarumi studies showed an improvement in exercise capacity and in patients with lung cancer and impaired pulmonary function, but are these improvements to reduce the postoperative respiratory complications or mortality of these patients? Probably this is the most important question to answer. Studies comparing postoperative results with and without prehabilitation could help us to answer this question.

In 2011 Benzo et al. (30) reported the results of two randomized single-blinded exploratory studies (prehabilitation $v s$. usual care) aimed to define a preoperative pulmonary rehabilitation intervention that may decrease the operative morbidity of curative lung cancer in patients with moderate-severe COPD. First randomized study tested 4 weeks of preoperative pulmonary rehabilitation. This study had very poor recruitment, mainly due to the fact that patients or providers were not willing to delay the curative surgery for 4 weeks. This study was finally stopped due to the low likelihood of meaningful accrual during the funding period. Nevertheless, no differences were found between the arms in any outcome for the 9 patients randomized. The problems with recruitment promote the need for changing prehabilitation program. And with this idea in the second randomized study patients in pulmonary rehabilitation group performed a ten-sessions of treatment in one week (twice a day). Nineteen patients were randomized to this study in one year, 9 to the control arm and 10 to the pulmonary rehabilitation arm. There were no statistical differences in patient demographic characteristic between groups. Patients in the prehabilitation arm had fewer days needing a chest tube (mean days of 4.7 vs. 9,0 $(\mathrm{P}=0.03)$ and a lower incidence of prolonged chest tubes (>7 days) compared to the controlled arm (11\% vs. 63\%, $\mathrm{P}=0.03$ ). Also, these patients had shorter length of stay in the hospital compared to patients in the control arm, but difference was not statistically significance (6.4 vs. 11.1 days, $\mathrm{P}=0.058)$. All these differences are crude estimators of postoperative morbidity and costs. However, these two randomized studies could only demonstrate the feasibility of the prehabilitation, mainly because the small size of patients randomized and the short prehabilitation training, just 1 week. These two items probably influence in lack of evidence of benefit of rehabilitation before lung resection in patients with impaired pulmonary function. 
Gao et al. (31) in 2015 published another study including 142 high-risk patients with potentially resectable lung cancer. Patients were distributed in a non-randomized way to a pre-operative pulmonary rehabilitation program followed by lobectomy, study group (71 patients), or underwent only lobectomy with conventional management, control group (71 patients). Respiratory training included training for abdominal breath, breath training device (volumetric exerciser) and lower extremity endurance training. The program for the intervention consisted in 2 sessions per day of 30-40 min during 3-7 days. The rate of postoperative complications in study group $(16.9 \%)$ was significantly lower than in group of control $(83.3 \%, \mathrm{P}=0.00)$. This statistical difference continued in postoperative lung infection $(2.81 \%$ in study group vs. $17.9 \%$ in control group, $\mathrm{P}=0.009)$. The postoperative stay was significantly longer in control group (7.21 vs. 11.07 days, $\mathrm{P}=0.00$ ), and an analysis of the average cost in hospital, including cost of pulmonary rehabilitation, showed no differences between two groups $(40,131.72$ vs. $36,943.33 ¥, \mathrm{P}=0.304)$. Gao concluded pre-operative pulmonary rehabilitation could improve cardiopulmonary function, control preoperative potential lung infection, decrease postoperative complications, and promote a faster recovery. All without an increment of total hospital stay or cost. However, some considerations about these results are needed. First, the criteria for high-risk patient. Smoking index $>800$ packyear and duration after quitting smoking $<2$ weeks, and bronchial hyperresponsiveness were two criteria to consider patients of high-risk and these patients represented near $60 \%(85 / 142)$ of selected patients to participate in the study. Only 57 (40,14\%) of 142 selected patients had criteria for impaired pulmonary function $(1.0 \mathrm{~L}<\mathrm{FEV} 1,40 \%<\mathrm{FEV} 1$ $<60 \%$, peak expiratory flow $<250 \mathrm{~L} / \mathrm{min} / \mathrm{kg}$ ). Also, some clinical characteristics were significantly different between study and control group, like TNM-stage or Histology. All these considerations together with the fact that the patients were not randomized make the results and conclusion of this study lose strength.

In 2017 the results of a randomized clinical trial carried out by Licker et al. (32) were published. One hundred fiftyone patients with operable lung cancer were randomly assigned to usual care $(\mathrm{n}=77)$ or preoperative rehabilitation $(\mathrm{n}=74)$. Prehabilitation training was based in a highintensity interval training (HIT) program with two to three sessions of 30 min per week during a median of 25 days. Maximal cardiopulmonary exercise testing and the six-minute walk test were performed twice before surgery.
Lung resections were performed by open thoracotomy or VATS. Respiratory function test, 6MWT and CPET were performed twice before surgery. The primary outcome measure was a composite of death and in-hospital postoperative complications. Secondary outcomes were the preoperative changes in CPET parameters and in 6MWT. The two group did not differ regarding preoperative demographic, clinical and functional data. More than $80 \%$ of patients underwent major lung resection through open thoracotomy. Peak oxygen consumption $\left(\right.$ peakVO $\left.\mathrm{K}_{2}\right)$ and six-minute walking distance increased $[+15 \%(\mathrm{P}=0.003)$ and $+15 \%(\mathrm{P}<0.001)]$ in the prehabilitation group whereas peakVO $\mathrm{V}_{2}$ declined in the usual care group $(-8 \% \mathrm{P}=0.005)$. No statistical differences were detected in postoperative complication between groups $35.5 \%$ in prehab group vs. $50.6 \%$ in usual care group, $\mathrm{P}=0.080$ ) or in length of hospital stay ( $9 v s .10$ days, $\mathrm{P}=0.223$ ). In the sub-analysis of pulmonary complications it was detected a lower incidence in the rehab group ( $23 \%$ vs. $44 \%, \mathrm{P}=0.018)$. Licker concluded they demonstrated the safety and effectiveness of a short-term exercise training program in improving aerobic performances in patients awaiting lung cancer surgery. However, these improvements failed to produce significant difference in composite morbidity-mortality index, compared with usual care. In this trial all patients with resectable lung cancer were included without taking in account the grade of postoperative complications risk (PFT and CPET), and this fact could had been one of the reasons to non-demonstrate statistical differences in postoperative complications between groups. Nevertheless, this could be a sign that not all patients could benefit from a prehabilitation program; a minimal lung function status (low-risk patients) could not justify the training intervention. On the other hand, a high number of patients were operated through open thoracotomy. Nowadays, VATS resection is considered first approach option to treat early stage of lung cancer, and actually almost all thoracic surgery centres follow this indication. In Licker series more than $80 \%$ of patients underwent lung resection through thoracotomy despite the fact that $48 \%$ of patients had a stage I lung cancer. This is another point to consider about the results.

Boujibar et al. (33) reported their results in a recent study with the objective to determine whether participation in a prehabilitation program would improve outcomes after surgery and lower morbidity according to the ClavienDindo classification. The cohort included 38 patients with potentially resectable lung cancer and $\mathrm{VO}_{2} \max$ $\leq 20 \mathrm{~mL} / \mathrm{min} / \mathrm{kg}$. Only minimal invasive surgery (VATS/ 
RATS) was used. Two groups were formed: one group with prehabilitation $(\mathrm{n}=19)$ and one group without prehabilitation $(\mathrm{n}=19)$. Four patients, all in the prehabilitation group, were excluded, 1 due to VATS conversion and 3 other because final treatment was chemotherapy/radiotherapy. The prehabilitation program consisted of exercise retraining, muscular strengthening of the lower and upper limbs, therapeutic education and help with smoking cessation. It was organized in 3 to 5 sessions per week until the operating date with duration of 90 minutes each session. There was no baseline difference between the two groups. The median prehabilitation sessions were 17 . The median number of days between CPET and surgery was 44, and no significant difference between the two groups was observed. The group of prehabilitation present lower postoperative complications ( $42 \%$ vs. $80 \%, \mathrm{P}=0.0382$ ) and the majority of these complications were a Clavien-Dindo grade of 2 or less, with statistically significant difference in favour of the prehabilitation group $(\mathrm{P}=0.0252)$. No differences between groups in terms of length of stay $(\mathrm{P}=0.644)$ were detected.

\section{Conclusions}

High risk of postoperative complications and mortality after a lung resection is a limitation for surgical treatment of lung cancer in patient with moderate-severe COPD. Different strategies intra-operatively (minimal invasive approach or protective ventilation) and post-operatively (analgesia control and physiotherapy) have reduce the morbi-mortality of these patients after lung resection, however, the incidence continue to be significant. Rehabilitation before surgery has demonstrated to reduce mortality and morbidity in colorectal, heart and spinal surgery, and this promoted the study of the role of prehabilitation in lung resection. The hypothesis about the effect of respiratory prehabilitation is that if we are able to improve respiratory function test and cardio-pulmonary exercise test (CPET) in highrisk patient secondary it could reduce the postoperative complications and mortality. Endurance training has been the preferred method to improve these parameters, but the long duration of this intervention (6 to 12 weeks) considering the oncological timing from the indication to surgery to the surgery day contraindicate the possibility to practice this training. High-intensity interval training has been postulated as the best alternative option to endurance training, with duration of the intervention from 2 to 6 weeks. It continues a limitation in some countries where patients can be operated in less than 2 weeks, but generally
4 weeks of waiting is the usual and scientific societies support these periods of time. Studies has demonstrated prehabilitation is safe and the improve respiratory functional test and cardiopulmonary exercise test in patients with resectable lung cancer and COPD using HIT for different period of time. However, the effect of these improvements in the postoperative complications and mortality has not been clarified. Some clinical trials have failed to demonstrate statistical difference but the non-randomization, patient's selection or short period of prehabilitation could justify these results. On the other hand, other clinical trials have showed a reduction of postoperative complication but the small number of patients included in the study and other limitations prevent generalize their results.

Licker study (32) seems to answer the question if all patients, with or without impaired lung function, could benefit from a prehabilitation program. Probably the effect of a prehabilitation in postoperative morbimortality in patients with correct lung function is limited and all efforts must be focused in COPD patients. Gao publication (31) studied the cost-effectiveness of a prehabilitation programme and find no statistical difference between prehab or usual care groups, the cost of prehabilitation was compensated by the shorter hospital stay and treatment of postoperative complications.

New randomized clinical trials with enough patients, correct duration of HIT ( 2 to 6 weeks) and focused in COPD patients are needed to clarify the suitability of prehabilitation. Meanwhile safety of prehabilitation and good results of some studies support this intervention in high-risk patients.

\section{Acknowledgements}

None.

\section{Footnote}

Conflicts of Interest: The authors have no conflicts of interest to declare.

\section{References}

1. Asamura H, Chansky K, Crowley J, et al. The International Association for the Study of Lung Cancer Lung Cancer Staging Project: Proposals for the Revision of the N Descriptors in the Forthcoming 8th Edition of the TNM Classification for Lung Cancer. J Thorac Oncol 
2015;10:1675-84.

2. Eberhardt WE, Mitchell A, Crowley J, et al. The IASLC Lung Cancer Staging Project: Proposals for the Revision of the M Descriptors in the Forthcoming Eighth Edition of the TNM Classification of Lung Cancer. J Thorac Oncol 2015;10:1515-22.

3. Nicholson AG, Chansky K, Crowley J, et al. The International Association for the Study of Lung Cancer Lung Cancer Staging Project: Proposals for the Revision of the Clinical and Pathologic Staging of Small Cell Lung Cancer in the Forthcoming Eighth Edition of the TNM Classification for Lung Cancer. J Thorac Oncol 2016;11:300-11.

4. Loganathan RS, Stover DE, Shi W, et al. Prevalence of COPD in women compared to men around the time of diagnosis of primary lung cancer. Chest 2006;129:1305-12.

5. Baser S, Shannon VR, Eapen GA, et al. Pulmonary dysfunction as a major cause of inoperability among patients with non-small-cell lung cancer. Clin Lung Cancer 2006;7:344-9.

6. Stanzani F, Paisani Dde M, Oliveira A, et al. Morbidity, mortality, and categorization of the risk of perioperative complications in lung cancer patients. J Bras Pneumol 2014;40:21-9.

7. Fan G, Filipczak L, Chow E. Symptom clusters in cancer patients: a review of the literature. Curr Oncol 2007;14:173-9.

8. Kenny PM, King MT, Viney RC, et al. Quality of life and survival in the 2 years after surgery for non small-cell lung cancer. J Clin Oncol 2008;26:233-41.

9. Edvardsen E, Skjonsberg OH, Holme I, et al. Highintensity training following lung cancer surgery: a randomised controlled trial. Thorax 2015;70:244-50.

10. Maeda K, Higashimoto Y, Honda N, et al. Effect of a postoperative outpatient pulmonary rehabilitation program on physical activity in patients who underwent pulmonary resection for lung cancer. Geriatr Gerontol Int 2016;16:550-5.

11. Mishra SI, Scherer RW, Snyder C, et al. Exercise interventions on health-related quality of life for people with cancer during active treatment. Cochrane Database Syst Rev 2012;(8):CD008465.

12. Brunelli A, Charloux A, Bolliger CT, et al. ERS/ESTS clinical guidelines on fitness for radical therapy in lung cancer patients (surgery and chemo-radiotherapy). Eur Respir J 2009;34:17-41.

13. Kehlet H. Fast-track colonic surgery: status and perspectives. Recent Results Cancer Res 2005;165:8-13.
14. Muehling BM, Halter GL, Schelzig H, et al. Reduction of postoperative pulmonary complications after lung surgery using a fast track clinical pathway. Eur J Cardiothorac Surg 2008;34:174-80.

15. Jones NL, Edmonds L, Ghosh S, et al. A review of enhanced recovery for thoracic anaesthesia and surgery. Anaesthesia 2013;68:179-89.

16. Society ATSER. Standards for the diagnosis and management of patients with COPD. 2014.

17. Fishman A, Martinez F, Naunheim K, et al. A randomized trial comparing lung-volume-reduction surgery with medical therapy for severe emphysema. N Engl J Med 2003;348:2059-73.

18. Wall BT, Dirks ML, van Loon LJ. Skeletal muscle atrophy during short-term disuse: implications for age-related sarcopenia. Ageing Res Rev 2013;12:898-906.

19. BTS recommendations to respiratory physicians for organising the care of patients with lung cancer. The Lung Cancer Working Party of the British Thoracic Society Standards of Care Committee. Thorax 1998;53 Suppl 1:S1-8.

20. Gibala MJ, McGee SL. Metabolic adaptations to shortterm high-intensity interval training: a little pain for a lot of gain? Exerc Sport Sci Rev 2008;36:58-63.

21. Burgomaster KA, Hughes SC, Heigenhauser GJ, et al. Six sessions of sprint interval training increases muscle oxidative potential and cycle endurance capacity in humans. J Appl Physiol (1985) 2005;98:1985-90.

22. Dunham C, Harms CA. Effects of high-intensity interval training on pulmonary function. Eur J Appl Physiol 2012;112:3061-8.

23. Hwang CL, Yu CJ, Shih JY, et al. Effects of exercise training on exercise capacity in patients with non-small cell lung cancer receiving targeted therapy. Support Care Cancer 2012;20:3169-77.

24. Wickerson L, Rozenberg D, Janaudis-Ferreira T, et al. Physical rehabilitation for lung transplant candidates and recipients: An evidence-informed clinical approach. World J Transplant 2016;6:517-31.

25. Rochester CL. Pulmonary rehabilitation for patients who undergo lung-volume-reduction surgery or lung transplantation. Respir Care 2008;53:1196-202.

26. Sekine Y, Chiyo M, Iwata T, et al. Perioperative rehabilitation and physiotherapy for lung cancer patients with chronic obstructive pulmonary disease. Jpn J Thorac Cardiovasc Surg 2005;53:237-43.

27. Jones LW, Peddle CJ, Eves ND, et al. Effects of presurgical exercise training on cardiorespiratory fitness 
among patients undergoing thoracic surgery for malignant lung lesions. Cancer 2007;110:590-8.

28. Bobbio A, Chetta A, Ampollini L, et al. Preoperative pulmonary rehabilitation in patients undergoing lung resection for non-small cell lung cancer. Eur J Cardiothorac Surg 2008;33:95-8.

29. Tarumi S, Yokomise H, Gotoh M, et al. Pulmonary rehabilitation during induction chemoradiotherapy for lung cancer improves pulmonary function. J Thorac Cardiovasc Surg 2015;149:569-73.

30. Benzo R, Wigle D, Novotny P, et al. Preoperative pulmonary rehabilitation before lung cancer resection: results from two randomized studies. Lung Cancer 2011;74:441-5.

Cite this article as: Sanchez-Lorente D, Navarro-Ripoll R, Guzman R, Moises J, Gimeno E, Boada M, Molins L. Prehabilitation in thoracic surgery. J Thorac Dis 2018;10(Suppl 22):S2593-S2600. doi: 10.21037/jtd.2018.08.18
31. Gao K, Yu PM, Su JH, et al. Cardiopulmonary exercise testing screening and pre-operative pulmonary rehabilitation reduce postoperative complications and improve fast-track recovery after lung cancer surgery: A study for 342 cases. Thorac Cancer 2015;6:443-9.

32. Licker M, Karenovics W, Diaper J, et al. Short-Term Preoperative High-Intensity Interval Training in Patients Awaiting Lung Cancer Surgery: A Randomized Controlled Trial. J Thorac Oncol 2017;12:323-33.

33. Boujibar F, Bonnevie T, Debeaumont D, et al. Impact of prehabilitation on morbidity and mortality after pulmonary lobectomy by minimally invasive surgery: a cohort study. J Thorac Dis 2018;10:2240-8. 